\title{
A NOTE ON NONUNITARY PRINCIPAL SERIES REPRESENTATIONS
}

\author{
HRVOJE KRALJEVIĆ
}

\begin{abstract}
It is proven in a rather elementary way that any nonunitary principal series representation of a semisimple Lie group $G$ is of finite length, having as a trivial consequence that the set of infinitesimal equivalence classes of quasisimple irreducible representations of $G$ with a given infinitesimal character is finite.
\end{abstract}

1. Introduction. The aim of this paper is to prove a theorem asserting that any nonunitary principal series representation of a semisimple Lie group $G$ is of finite length. This theorem has been known already for a long time. However, its proof was based on the fact that the set of infinitesimal equivalence classes of irreducible representations with a given infinitesimal character is finite. This latter fact was proven using a very deep result of Harish-Chandra- that all invariant eigendistributions on $G$ are representable by locally integrable functions on $G$.

In [4] the above-mentioned theorem was proven in a more elementary way in the case that $G$ is linear, but the proof does not generalize to nonlinear groups. The authors use rather careful investigation of finite dimensional representations of $G$, and Kostant's (or, more elementary, Helgason's) result on cyclicity of a $K$-fixed vector in some spherical nonunitary principal series representations.

Our method to prove the theorem (for general $G$ ) is similar but seems even simpler, because it does not use such fine results on finite dimensional representations. Also, instead of Helgason's result on cyclicity, we use a more elementary theorem of Miličić.

2. Notation. For any Lie group $H$ we denote by $\hat{H}$ the set of equivalence classes of irreducible finite dimensional continuous representations of $H$ on complex vector spaces. We shall usually not distinguish between a representation and its equivalence class. If $\sigma$ is a finite dimensional continuous representation of $H, \tilde{\sigma}$ will stand for the contragredient representation.

If $S$ is a subset of a complex vector space, span $S$ will denote the subspace spanned by $S$. The subscript " $c$ " will denote the complexification of a real vector space.

Received by editors July 6, 1977.

AMS (MOS) subject classifications (1970). Primary 22E45.

Key words and phrases. Semisimple Lie groups, nonunitary principal series.

(1) American Mathematical Society 1978 
3. The main result. Let $G$ be a connected semisimple Lie group with Lie algebra $\mathfrak{g}, G=K A N$ an Iwasawa decomposition of $G$ and $\mathfrak{g}=\mathfrak{f}+\mathfrak{a}+\mathfrak{n}$ the corresponding decomposition of g. Let $M$ be the centralizer of $A$ in $K$. Then $P=M A N$ is a closed subgroup of $G-$ a minimal parabolic subgroup.

Let $\mathcal{G}$ be the universal enveloping algebra of $g_{c}$.

Let $\sigma$ be a continuous representation of $P$ on a finite dimensional complex space $V$. Denote by $V^{\sigma}$ the space of all functions $f \in C^{\infty}(G, V)$ with the following properties:

(i) $f(p x)=\sigma(p) f(x), p \in P, x \in G$;

(ii) $\operatorname{dim} \operatorname{span}\left\{f^{k} ; k \in K\right\}<\infty$, where $f^{k}(x)=f(x k), x \in G$. For $X \in \mathfrak{g}$ and $f \in V^{\sigma}$ let $\pi^{\sigma}(X) f: G-, V$ be defined by

$$
\left(\pi^{\sigma}(X) f\right)(x)=(d / d t)_{t=0} f(x \exp t X), \quad x \in G .
$$

Then $\pi^{\sigma}(X) f \in V^{\sigma}$ and $\pi^{\sigma}: X \mapsto \pi^{\sigma}(X)$ is a representation of $\mathfrak{g}$ on $V^{\sigma} . \pi^{\sigma}$ extends to $\mathcal{G}$, hence $V^{\circ}$ becomes a $\mathcal{G}$-module. It is also a $K$-module under the right translation of functions.

If $\tau$ is a finite dimensional representation of $M$ on $V$ and $\lambda \in \mathrm{a}_{c}^{*}$, we define the representation $(\tau, \lambda)$ of $P$ on $V$ by

$$
(\tau, \lambda)(\text { man })=e^{\lambda(\log a)} \tau(m), \quad m \in M, a \in A, n \in N .
$$

We have then $\hat{P}=\left\{(\tau, \lambda) ; \tau \in \hat{M}, \lambda \in a_{c}^{*}\right\} . V^{(\tau, \lambda)}, \tau \in \hat{M}, \lambda \in a_{c}^{*}$, are called nonunitary principal series $\mathcal{G}$-modules.

THEOREM. Every nonunitary principal series $\mathcal{G}$-module is of finite length.

Proof. Let $\Sigma$ be the restricted root system of $g$ in $a^{*} \subset a_{c}^{*}$. The chosen Iwasawa decomposition fixes the set $\Sigma^{+}$of positive restricted roots. For any $\alpha \in \Sigma$ let $\mathfrak{g}^{\alpha}$ be the corresponding root subspace of $\mathfrak{g}$ and let $H_{\alpha}$ be the unique element of $a \cap\left[\mathfrak{g}^{\alpha}, \mathfrak{g}^{-\alpha}\right]$ such that $\alpha\left(H_{\alpha}\right)=2$. Furthermore, set

$$
\rho=\frac{1}{2} \sum_{\alpha \in \Sigma^{+}}\left(\operatorname{dim} \mathrm{g}^{\alpha}\right) \cdot \alpha .
$$

For $\gamma \in \hat{G}$ realized on $W$ let $\lambda_{\gamma}$ denote the lowest restricted weight (with respect to $\Sigma^{+}$) of the g-module $W$. Let $W^{-}$be the corresponding restricted weight subspace of $W$ and let $W^{+}$be the sum of all the other restricted weight subspaces. Then $W^{-}$is invariant and irreducible under $M$ and we denote by $\tau_{\gamma}$ the corresponding element of $\hat{M} . W^{+}$is a $P$-submodule of $W$ and the representation of $P$ on $W / W^{+}$is $\left(\tau_{\gamma}, \lambda_{\gamma}\right)$. Hence, $\left(\tau_{\gamma}, \lambda_{\gamma}\right)$ is a quotient of the representation $\gamma \mid P$. $\lambda_{\gamma}$ assumes real values on $\mathfrak{a}$ and $\lambda_{\gamma}\left(H_{\alpha}\right) \leqslant$ 0 for any $\alpha \in \Sigma^{+}$. Furthermore, for any $\mu \in a^{*}$ there exists $\gamma \in \hat{G}$ such that $\lambda_{\gamma}\left(H_{\alpha}\right) \leqslant \mu\left(H_{\alpha}\right)$ for every $\alpha \in \Sigma^{+}$.

Let $\omega \in \hat{M}$ and $\nu \in \mathrm{a}_{c}^{*}$. We want to prove first that the $\mathcal{G}$-module $V^{(\omega, \nu)}$ is finitely generated.

Choose $\gamma \in \hat{G}$ so that $\lambda_{\gamma}\left(H_{\alpha}\right)<\operatorname{Re} \nu\left(H_{\alpha}\right)-\rho\left(H_{\alpha}\right)$ for any $\alpha \in \Sigma^{+}$. Set $\lambda=\nu-\lambda_{\gamma}$. Let $\sigma \in \hat{M}$ be contragredient to some irreducible constituent of $\tau_{\gamma} \otimes \tilde{\omega}$. Then $\sigma \otimes \tau_{\gamma} \otimes \tilde{\omega}$ fixes a nonzero vector, hence $\omega$ is a direct summand 
in $\sigma \otimes \tau_{\gamma} \cdot\left(\tau_{\gamma}, \lambda_{\gamma}\right)$ being a quotient of $\gamma \mid P$, it follows that $(\omega, \nu)=\left(\omega, \lambda+\lambda_{\gamma}\right)$ is a quotient of $(\sigma, \lambda) \otimes(\gamma \mid P)$. Let $W$ be the space of $\gamma$. By Proposition (10.6) in [2], there exists a surjective $\mathcal{G}$-morphism of $V^{(\sigma, \lambda)} \otimes W$ onto $V^{(\omega, \nu)}$. If we prove that $V^{(\sigma, \lambda)}$ is finitely generated, Proposition 3.3 in [3] will imply that $V^{(\sigma, \lambda)} \otimes W$, hence also $V^{(\omega, \nu)}$, is finitely generated.

Now, $\operatorname{Re}(2 \rho-\lambda)\left(H_{\alpha}\right)=\operatorname{Re}\left(2 \rho-\nu+\lambda_{\gamma}\right)\left(H_{\alpha}\right)<\rho\left(H_{\alpha}\right)$ for every $\alpha \in \Sigma^{+}$. By Theorem 3.1 in [6], $V^{(\tilde{\sigma}, 2 \rho-\lambda)}$ contains the smallest $\mathcal{G}$-submodule different from $\{0\}$. There exists a canonical $\mathcal{G}$-invariant (and $K$-invariant) nondegenerate bilinear form on $V^{(\tilde{\sigma}, 2 \rho-\lambda)} \times V^{(\sigma, \lambda)}$ (see e.g. Lemma 5.1 in [4]), hence $V^{(\sigma, \lambda)}$ contains a proper $\mathcal{G}$-submodule $U$ which contains any other proper $\mathcal{G}$-submodule of $V^{(\sigma, \lambda)}$. But then every vector in $V^{(\sigma, \lambda)}$ not contained in $U$ generates the $\mathcal{G}$-module $V^{(\sigma, \lambda)}$. Especially, $V^{(\sigma, \lambda)}$ is finitely generated.

Thus, we have proved that every nonunitary principal series module is finitely generated. $\mathcal{G}$ being Noetherian, all of these modules are Noetherian. By the above mentioned canonical pairing on $V^{(\tilde{\omega}, 2 \rho-\nu)} \times V^{(\omega, \nu)}$ all of these modules are also Artinian. Hence, they are of finite length. Q.E.D.

\section{Consequences and remarks.}

COROLlary. Suppose that $G$ has finite center. Let $\mathscr{Z}$ be the center of $\mathcal{G}$ and $\chi \in \operatorname{Hom}(\mathscr{Z}, \mathbf{C})$. There exist only finitely many equivalence classes of irreducible $(\mathcal{G}, K)$-modules with infinitesimal character $\chi$.

Proof. By Casselman's theorem [1], [5], [6] every irreducible ( $\mathcal{G}, K$ )-module is equivalent to a submodule of some $V^{(\sigma, \lambda)}, \sigma \in \hat{M}, \lambda \in \mathrm{a}_{c}^{*}$. The number of pairs $(\sigma, \lambda) \in \hat{M} \times a_{c}^{*}$, such that $V^{(\sigma, \lambda)}$ has infinitesimal character $\chi$, being obviously finite, the Corollary follows immediately from the Theorem. Q.E.D.

Remark 1. It is easy to generalize the Theorem to the case of a reductive Lie group $G$ such that $Z G^{0}$ is of finite index in $G$, where $Z$ is the center and $G^{0}$ is the identity component of $G$. In this case nonunitary principal series modules are $(\mathcal{G}, K)$-modules induced by finite dimensional irreducible representations of $P$, where $P$ is the normalizer of a minimal parabolic subalgebra of $[\mathfrak{g}, \mathfrak{g}]$ and $K$ is the centralizer in $G$ of a Cartan involution on $[\mathfrak{g}, \mathfrak{g}]$.

ReMARK 2. Let $G$ be a group in the Harish-Chandra class, $Q$ any parabolic subgroup of $G, N$ the unipotent radical of $Q, L$ a Levi factor of $Q$. Let $\sigma$ be an irreducible quasisimple representation of $L$ extended to the representation of $P$ (trivial on $N$ ). By Casselman's theorem [1] $\sigma$ is (infinitesimally) equivalent to a subrepresentation of some nonunitary principal series representation of $L$. An induction in stages argument shows that the induced representation $\pi^{\sigma}$ of $G$ is equivalent to a subrepresentation of some nonunitary principal series representation of $G$. Especially, $\pi^{\sigma}$ is of finite length.

I express my thanks to D. Miličić for his interest, for showing me his manuscript [5], and for calling my attention to Proposition (10.6) in [2] which shortened the original version of this paper. 


\section{REFERENCES}

1. W. Casselman, The differential equations satisfied by matrix coefficients, 1975 (manuscript).

2. A. W. Knapp and N. R. Wallach, Szego kernels associated with discrete series, Invent. Math. 34 (1976), 163-200.

3. B. Kostant, On the tensor product of a finite and an infinite dimensional representation, J. Functional Analysis 20 (1975), 257-285.

4. J. I. Lepowsky and N. R. Wallach, Finite- and infinite-dimensional representations of linear semisimple groups, Trans. Amer. Math. Soc. 184 (1973), 223-246.

5. D. Miličic, Notes on asymptotics of admissible representations, 1976 (unpublished).

6. __ Asymptotic behaviour of matrix coefficients of the discrete series, Duke Math. J. 44 (1977), 59-88.

Department of Mathematics, University of ZAGreb, Zagreb, Yugoslavia

Current .àddress: Nova Ves 86, 41000 Zagreb, Yugoslavia 